\title{
Ginsenosides: A Potential Neuroprotective Agent
}

\author{
Mengmeng Zheng, ${ }^{1}$ Yizhou Xin, ${ }^{2}$ Yujuan Li, ${ }^{1}$ Fangxue Xu, ${ }^{1}$ Xiaozhi Xi, ${ }^{1}$ Hong Guo, \\ Xiaowei Cui, ${ }^{1}$ Hui Cao, ${ }^{1}$ Xi Zhang, ${ }^{1}$ and Chunchao Han ${ }^{10}{ }^{1}$ \\ ${ }^{1}$ School of Pharmacy, Shandong University of Traditional Chinese Medicine, Jinan 250355, China \\ ${ }^{2}$ The Affiliated Hospital of Shandong University of Traditional Chinese Medicine, Jinan 250011, China \\ Correspondence should be addressed to Chunchao Han; chunchaoh@126.com
}

Received 6 December 2017; Revised 6 March 2018; Accepted 2 April 2018; Published 8 May 2018

Academic Editor: Mai S. Li

Copyright (C) 2018 Mengmeng Zheng et al. This is an open access article distributed under the Creative Commons Attribution License, which permits unrestricted use, distribution, and reproduction in any medium, provided the original work is properly cited.

Ginseng is a traditional Chinese medicine with a wide range of pharmacological activities. Ginsenosides are the major constituents of ginseng. Ginsenosides have the unique biological activity and medicinal value, such as antitumor, anti-inflammatory, antioxidation, and inhibition of cell apoptosis. With the increase of stress in life, the incidence of nervous system diseases is also increasing. Neurological diseases pose a huge burden on people's life and health. In recent years, some studies have shown that ginsenosides have a certain role in the prevention and treatment of neurological diseases. However, the research is still in its infancy, and the relevant mechanisms are complex. In the paper, we review the effects and mechanisms of ginsenosides on epilepsy, depression, cerebral ischemia reperfusion injury, Alzheimer's disease, and Parkinson's disease. We hope to provide a theoretical basis for the treatment of nervous system diseases by ginsenosides.

\section{Introduction}

Ginseng is the traditional valuable medicinal herb that has been widely used in China for thousands of years. Ginseng is used as energy booster, and it can fortify the spleen to benefit the lungs, nourish fluids, calm the heart, tranquilize the mind, and so on [1-3]. Ginseng has such a good effect, mainly due to ginsenosides. Ginsenosides are the major active ingredients of ginseng and are extracted from roots, fruits, stems, and leaves of ginseng. At present, more than 60 ginsenosides were isolated and identified from Araliaceae family. The basic structure of ginsenosides is similar because almost all ginsenosides contain 30 carbon atoms, and they are arranged in four rings of steroid nuclei [4]. According to the difference in the position and quantity of sugar moiety, ginsenosides are divided into three types (Figure 1): A-Panaxadiol group (e.g., Rb1, Rb2, Rb3, Rc, Rd, Rg3, and Rh2), B-Panaxatriol group (e.g., Re, Rg1, Rg2, and Rh1), and C-Oleanolic acid group (e.g., Ro) [5, 6]. Each ginsenoside plays a different pharmacological role. Modern pharmacological experiments have shown that ginsenosides have many biological activities. For example, ginsenosides have good effects in anticancer, anti-inflammation, antioxidation, antiaging, antifatigue, and physiological functions [7-10].

The main features of nervous system disease are sensation, movement, consciousness, and autonomic nervous dysfunction. Common neurological disorders include Alzheimer's disease, Parkinson's disease, epilepsy, and depression. Nervous system diseases have a great impact on the life and work of the patients and can even lead to life-threatening situations. Nervous system diseases have become another major disease after heart disease and cancer. With the increase of aging population and the pressure of life, the prevalence of nervous system diseases will become higher and higher. Therefore, it is very meaningful to study and treat nervous system diseases. Recently, ginsenosides are becoming more and more concerned in the treatment of neurological diseases. Some studies have shown that ginsenosides do have good preventive and therapeutic effect on neurological diseases [11, 12]. For instance, ginsenoside $\mathrm{Rg} 2$ protects against memory impairment via antiapoptosis in rat models with vascular dementia [13]. The neuroprotective mechanisms of ginsenosides include antioxidant effect, antiapoptosis effect, estrogen-like effect, 


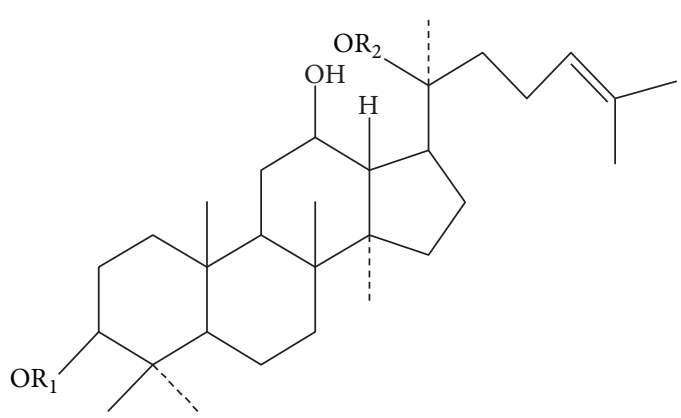

\begin{tabular}{lll} 
A-Panaxadiol group & & \\
\hline & $\mathrm{R} 1$ & $\mathrm{R} 2$ \\
\hline 20(S)-protopanaxadiol & $\mathrm{H}$ & $\mathrm{H}$ \\
$\mathrm{Rb} 1$ & $\mathrm{Glc}(2 \rightarrow 1) \mathrm{Glc}$ & $\mathrm{Glc}(6 \rightarrow 1) \mathrm{Glc}$ \\
$\mathrm{Rb} 2$ & $\mathrm{Glc}(2 \rightarrow 1) \mathrm{Glc}$ & $\mathrm{Glc}(6 \rightarrow 1) \mathrm{Ara}(\mathrm{p})$ \\
$\mathrm{Rc}$ & $\mathrm{Glc}(2 \rightarrow 1) \mathrm{Glc}$ & $\mathrm{Glc}(6 \rightarrow 1) \mathrm{Ara}(\mathrm{f})$ \\
$\mathrm{Rd}$ & $\mathrm{Glc}(2 \rightarrow 1) \mathrm{Glc}$ & $\mathrm{Glc}$ \\
Rg3 & $\mathrm{Glc}(2 \rightarrow 1) \mathrm{Glc}$ & $\mathrm{H}$ \\
$\mathrm{Rh} 2$ & $\mathrm{Glc}$ & $\mathrm{H}$
\end{tabular}

B-Panaxatriol group

\begin{tabular}{lll}
\hline & R1 & R2 \\
\hline 20(S)-protopanaxtriol & H & H \\
Re & Glc $(2 \rightarrow 1)$ Rha & Glc \\
Rg1 & Glc & Glc \\
Rg2 & Glc $(2 \rightarrow 1)$ Glc & H \\
Rh1 & Glc & H \\
\hline
\end{tabular}<smiles>CCCCC[C@@H](O)C1CC2(C)C3CCC(C(C)(C)CCC=C(C)C)C3C(O)CC2C2(C)CCC(O)C(C)(C)C12</smiles>

C-Oleanolic acid group

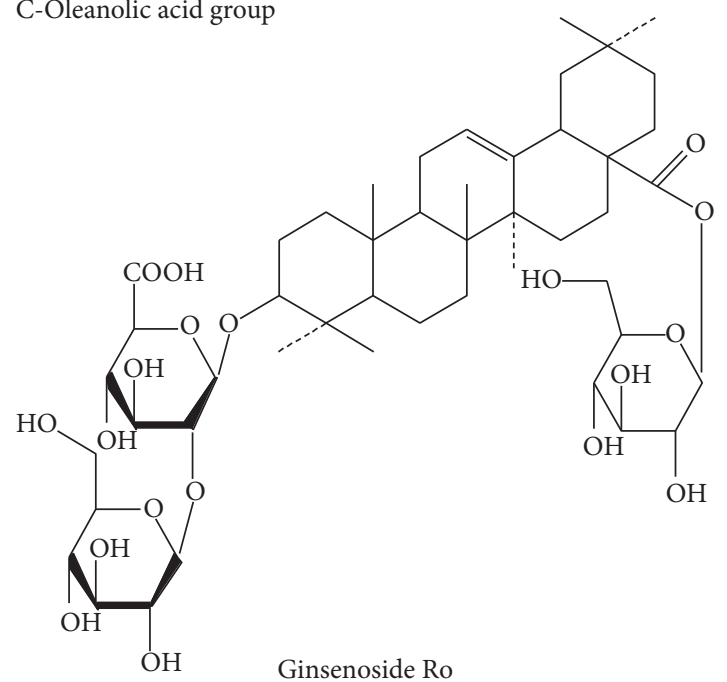

Figure 1: Chemical structure of Panaxadiol, Panaxatriol, and Oleanolic acid group.

restraining the influence of nitric oxide and nitric oxide synthase, and improving mitochondrial dysfunction [14].

At present, the treatment of nervous system diseases is mainly drugs and surgical treatment. However, patients often cannot adhere to the formal medication. In addition, long-term use of drugs can lead to drug accumulation and poisoning. Surgical treatment often increases the chance of infection and leads to other dysfunctions. In recent years, western countries have been using natural medicine to treat nervous system diseases, which is considered to be promising research method [15]. Ginsenoside is one of the most valuable natural medicines. Animal experiments have shown that ginsenosides are effective in treating nervous system diseases, such as Parkinson's and Alzheimer's disease (Figure 2). In the paper, we reviewed the mechanism of ginsenosides in Parkinson's disease, Alzheimer's disease, epilepsy, cerebral ischemia reperfusion injury, and depression in order to provide assistance for the treatment of nervous system diseases by ginsenosides and stimulate more research to examine the clinical efficacy of ginsenosides (Figure 3). 


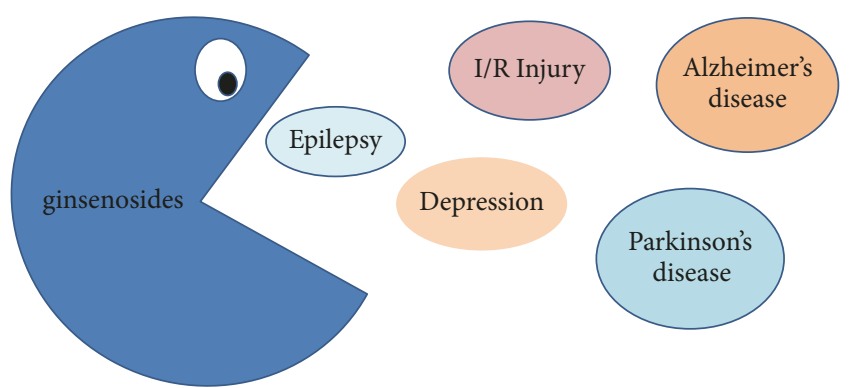

Figure 2: The effect of ginsensides on nervous system disease, mainly including epilepsy, depression, cerebral ischemia reperfusion injury, Alzheimer's disease, and Parkinson's disease.

\section{The Pharmacological Activity and Mechanism of Ginsenosides in Nervous System Diseases}

2.1. Antiepilepsy Effect. Epilepsy is a kind of chronic brain disease caused by abnormal discharge of neurons in the brain, which is characterized by recurrent seizures [16]. There are about 65 million people with epilepsy in the world, and 2.4 million new cases occur every year [17]. Clinically, the treatment of epilepsy is mainly antiepileptic drugs, but antiepileptic drugs still cannot effectively control many epileptic patients. Antiepileptic drugs also could lead to death, neurological disorders, and other adverse reactions. Therefore, it is meaningful to find new antiepileptic drugs. Happily, natural products usually have less toxic and side effects. Ginsenosides are the main active components of ginseng and have a good antiepileptic effect.

Lian et al. have compared the anticonvulsant activity of whole root extract, whole leaves/stems extract, and Rb extract. Three seizure models were prepared by kainic acid (KA), pilocarpine, and pentylenetetrazol (PTZ), respectively. The results showed that $\mathrm{Rb}$ extract had obvious anticonvulsant effect and was dose-dependent in three models. The Rb extract not only could increase the latency to the seizures, but also could decrease the seizure score, weight loss, the seizure duration, and neuronal damage. The above results suggest that panaxadiol group has a good effect on nervous system, because the $\mathrm{Rb}$ component belongs to panaxadiol group [18]. Compared with the $\mathrm{Rb}$ extract, the root extract and the leaves/stems extract had no significant anticonvulsant effect. This hints that some ginsenosides have anticonvulsant effects, while some ginsenosides do not have anticonvulsant effects. Lian et al. also compared the anticonvulsant effects of ginsenosides Rb1, Rb3, Rd, and mixtures of them. The results suggest that the mixtures of purified ginsenosides $\mathrm{Rb} 1, \mathrm{Rb} 3$ with or without Rd has significant anticonvulsant effects in three models induced by KA, PTZ, and pilocarpine. Amusing, no one individual ginsenoside accounted for the activity of the $\mathrm{Rb}$ extract. Therefore, it is of great significance to explore the combination of ginsenosides for the development of anticonvulsant drugs. Furthermore, studies have shown that ginsenoside $\mathrm{Rb} 2$ plays an important role in anticonvulsant activity [19]. These results suggest that the $\mathrm{Rb}$ fraction has the potential to become anticonvulsant drug.

Intracellular calcium concentration $\mathrm{Ca}^{2+}$ is an important indicator of neurological disorder. The level of $\mathrm{Ca}^{2+}$ would increase in epilepsy. Total ginsenosides and ginsenoside $\mathrm{Rg} 3$ could inhibit the increase of $\mathrm{Ca}^{2+}$ induced by $\mathrm{Mg}^{2+}$. Ginsenosides could modulate perturbed homeostasis of $\mathrm{Ca}^{2+}$ by the inhibition of N-methyl-D-aspartate receptor [20]. Besides, oxidative stress could lead to hippocampal degeneration. It is interesting to note that ginsenosides attenuated oxidative stress in the synaptosome and reduced synaptic vesicles at the presynaptic terminals dose dependently. What is more, not adenosine $A_{1}$ receptor or adenosine $A_{2 B}$ receptor, but adenosine $A_{2 A}$ receptors play an important role in antiepilepsy. Therefore, ginsenosides exert antiepilepsy effect by activating adenosine $\mathrm{A}_{2 \mathrm{~A}}$ receptors [21] (Table 1).

2.2. Antidepressant-Like Effect. Depression is neuropsychiatric disorder characterized by sleep disturbances, low selfesteem, guilty feelings, and suicidal tendencies [22-24]. Depression can reduce the body weight and appetite of patients [25]. About 15\% people with depression died from suicide. According to the World Health Organization, depression has become the second major disease burden in China. Clinically, monoamine oxidase inhibitors are commonly used to treat depression [26]. The mechanism of antidepressant drugs may be related to proteins, neurotransmitters, hormones, energy sources, and minerals [27]. Nevertheless, antidepressant drugs would cause irreversible side effects. Therefore, it is necessary to find a safe and effective medicine for treating depression. It is wise to look for active ingredients from natural products.

In order to explore the antidepressant effect of ginsenoside Rb3, some tests and biochemical changes were carried out. Researchers found that ginsenoside Rb3 could significantly reduce immobility time in forced swim test and tail suspension test. In addition, ginsenoside Rb3 decreased the number of escape failures in the learned helplessness procedure. Besides, ginsenoside Rb3 not only increased sucrose preference, locomotor activity, and novelty-suppressed of mice, but also attenuated hypothermia, palpebral ptosis, and akinesia. Furthermore, ginsenoside Rb3 would reverse the decrease of hippocampal weight and the brain-derived neurotrophic factor (BDNF) level in the hippocampus [28]. As we all known, substances can be hydrolyzed in the intestine. In order to explore substances that exert antidepressant activity, ginsenoside Rb3 and its four-deglycosylated derivatives (ginsenoside Rg3, ginsenoside Rh2, compound $\mathrm{K}$, and 20(S)-protopanaxadiol (PPD)) were tested in depression mice model. The results indicated that ginsenoside $\mathrm{Rg} 3$ had better antidepressant effect than compound $\mathrm{K}$, ginsenoside Rb3, ginsenoside Rh2, and PPD. Ginsenoside Rg3 exerted antidepressant effect by regulating noradrenaline (NA), adrenocorticotropic hormone (ACTH), and corticosterone (CORT) levels in the brain region of mice. Moreover, the effects of compound $\mathrm{K}$ and ginsenoside Rb3 were similar, but ginsenoside Rh2 and PPD failed to show any effect [29]. In addition, ginsenoside Rg3 also exerted antidepressant 


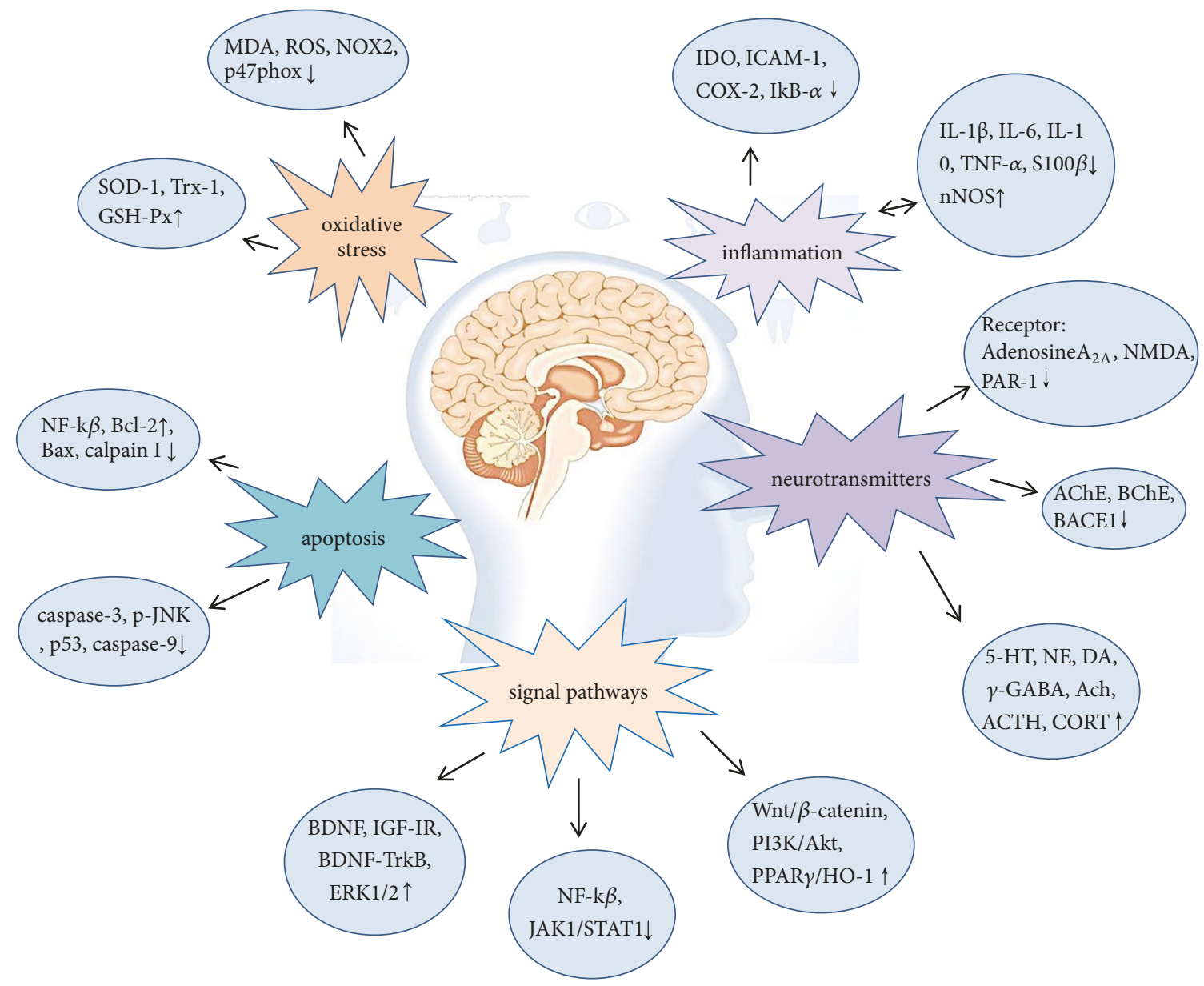

FIgURE 3: Several neuroprotection mechanisms of ginsenosides.

effect through promoting the hippocampal BDNF signaling pathway [30].

The antidepressant effect of ginsenoside Rg1 was explored in a mice model of depression and a mouse model of chronic mild stress (CMS) depression. Changes were investigated in hippocampal neurogenesis, spine density, BDNF signaling pathway, and serum corticosterone level. The results suggested that ginsenoside Rg1 reversed the decrease of dendritic spine density and hippocampal neurogenesis. Moreover, ginsenoside Rgl exerted antidepressant effect by activating the BDNF signaling pathway and upregulating hippocampal neurogenesis [31]. In addition, ginsenoside Rgl could increase protein kinase A (PKA) and cAMP-response element binding protein (CREB) phosphorylation levels. And ginsenoside Rg1 increased BDNF expression in the amygdala of rats [32]. These results suggest that ginsenoside Rb1 is a very promising antidepressant. Moreover, depression-like and anxiety-like behaviors and cognition memory deficit were prepared by repeated immobilization. Ginsenoside Re could decrease the level of tyrosine hydroxylase (TH) expression in the locus coeruleus and increase BDNF mRNA expression in the hippocampus. What is more, ginsenoside Re significantly restored body weight and serum corticosterone (CORT) level and blocked the increase of corticotrophin-releasing factor (CRF) in the hypothalamus [33]. So, ginsenoside Re inhibited the stress-induced behavioral deficits by modulating the central noradrenergic system. It is well known that menopausal women are more likely to suffer from depression and the depression is more severe. The findings indicated that ginsenoside $\mathrm{Rb}$ and compound $\mathrm{K}$ significantly prevented ovariectomy-induced prolongation of immobility, whereas PPD, ginsenosides Rg1, and Ro did not have any significant effect on immobility time. In addition, coadministration of ginsenoside $\mathrm{Rb} 1$ and ritanserin, $5-\mathrm{HT}_{2 \mathrm{~A}}$ receptor antagonist, did not reduce the immobility time in ovariectomized mice, because ritanserin antagonized the effect of ginsenoside Rb1. Therefore, ginsenoside $\mathrm{Rb} 1$ activated $5-\mathrm{HT}_{2 \mathrm{~A}}$ receptor to exert antidepressant effect [34].

Antidepression effect of St John's Wort extract (SJ), ginsenosides, and clomipramine (CPM) were observed in chronic unpredictable mild stress-induced depression by using a combination of behavioral assessments and metabonomics. Ginsenosides could increase the activity frequency of model animals, while SJ and CPM had only a mild effect. Besides, ginsenosides not only significantly increased food consumption and body weight, but also alleviated the reduction of adrenal and thymus indices. Furthermore, ginsenosides significantly increased plasma ACTH and serum CORT 
TABLE 1: Pharmacological activity and mechanism of ginsenosides on epilepsy, depression, and cerebral ischemia reperfusion injury.

\begin{tabular}{|c|c|c|c|}
\hline Pharmacological effects & Ginsenosides & Mechanism & References \\
\hline \multirow[t]{2}{*}{ Antiepilepsy } & Total ginsenosides & Adenosine $\mathrm{A}_{2 \mathrm{~A}}$ receptor $\downarrow$ & [21] \\
\hline & $\mathrm{Rg} 3$ & $\begin{array}{l}\text { NMDA receptor-mediated } \\
\qquad \mathrm{Ca}^{2+} \downarrow\end{array}$ & {$[20]$} \\
\hline \multirow[t]{7}{*}{ Antidepressant-like effects } & Rg1 & $\begin{array}{l}\text { PKA and CREB phosphorylation, } \\
\text { the expression of BDNF, hippocampal neurogenesis } \uparrow\end{array}$ & {$[31,32]$} \\
\hline & Total ginsenosides & $\begin{array}{c}\text { IL- } 1 \beta, \text { IL- } 6, \text { TNF- } \alpha \text {, IDO } \downarrow \\
\text { ACTH, CORT } \uparrow\end{array}$ & {$[35,36]$} \\
\hline & $\mathrm{Rb} 1$ & $5-\mathrm{HT}_{2 \mathrm{~A}}$ receptors $\uparrow$ & {$[34]$} \\
\hline & $\mathrm{Rb} 3$ & BDNF levels $\uparrow 5$-HT $\uparrow \downarrow$ & [28] \\
\hline & $\operatorname{Re}$ & $\begin{array}{c}\text { BDNF mRNA expression } \uparrow \\
\text { TH, CRF } \downarrow\end{array}$ & {$[27]$} \\
\hline & $\mathrm{CK}$ & $\begin{array}{c}5-\mathrm{HT}_{2 \mathrm{~A}} \text { receptors, NA } \uparrow \\
\mathrm{ACTH} \text {, corticosterone level } \downarrow\end{array}$ & {$[29,34]$} \\
\hline & $\operatorname{Rg} 3$ & $\begin{array}{c}\text { BDNF signaling pathway, NA } \uparrow \\
\text { ACTH, corticosterone level } \downarrow\end{array}$ & {$[29,30]$} \\
\hline \multirow[t]{5}{*}{$\begin{array}{l}\text { Protection against cerebral } \\
\text { ischemia reperfusion injury }\end{array}$} & Rg1 & $\begin{array}{c}\text { Bcl-2, Trx-1, SOD-1, PKB/Akt, } \\
\text { p-NF-kB p65, HSP70 } \uparrow \\
\text { Bax, aquaporin } 4 \text { expression, IL-1 } \beta \text {, TNF- } \alpha \text {, HMGB1, cleaved } \\
\text { caspase-3, cleaved caspase-9, RAGE, PAR-1, } \\
\text { Ca }^{2+} \text { overload, NF-kB, } \\
\text { JAK1/STAT1 signal pathways } \downarrow\end{array}$ & {$[37-42]$} \\
\hline & $\mathrm{Rb} 1$ & $\begin{array}{c}\text { MDA, NF-k } \beta, \\
\text { JAK1/STAT1 signal pathways, } \\
\text { TNF- } \alpha \text {, IL-6, LC3, Beclin } 1 \downarrow \text { Trx-1, SOD-1, PKB/Akt, HSP70 } \uparrow\end{array}$ & {$[42-45]$} \\
\hline & $\mathrm{Rd}$ & $\begin{array}{c}\text { VEGF, BDNF, } \\
\text { PI3K/Akt, ERK1/2 pathways } \uparrow \\
\text { phosphorylation of NMDAR 2B subunit } \downarrow\end{array}$ & {$[46,47]$} \\
\hline & $\mathrm{Re}$ & MDA $\downarrow \mathrm{H}^{+}$-ATPase $\uparrow$ & {$[48]$} \\
\hline & Rg3 & Calpain I, caspase-3 $\downarrow \downarrow$ & {$[49]$} \\
\hline
\end{tabular}

levels in model rats [35]. Kang et al. came up with a new idea that the antidepression effect of total ginsenosides may be associated with its anti-inflammation. The results suggested that ginsenosides would attenuate LPS-induced depressionlike behavior in forced swimming test, tail suspension test, and sucrose preference test. On the one hand, total ginsenosides decreased 5-HT level, tryptophane turnover, and indoleamine 2, 3-dioxygenase (IDO) activities in brain. On the other hand, ginsenosides reduced the levels of IL- $1 \beta$, IL6 , TNF- $\alpha$, and IDO in hippocampus. Therefore, studying the peripheral anti-inflammatory effects of ginsenosides may contribute to treatment of neurological diseases [36] (Table 1).

\subsection{Protection against Cerebral Ischemia Reperfusion Injury.} Acute cerebral ischemia can cause serious nervous system and motor impairment, threatening human life and health. It has become an important disease, which endangers the health of the elderly in modern society. Cerebral ischemia reperfusion injury can lead to apoptosis and necrosis in the ischemic and peripheral nerve cells. Cerebral ischemia reperfusion injury may be the underlying pathological cause of stroke, which is one of leading causes of death and acquired disability in the world $[69,70]$. In addition, the incidence of stroke is higher in developing countries [71]. The high mortality and disability rates of ischemic stroke are a huge burden on society and family. There are few methods to treat ischemia reperfusion injury, and the mechanisms involved are very intricate. Ginsenosides play an important role in the treatment of cerebral ischemia reperfusion injury. Some studies have explored the mechanism of ginsenosides in the treatment of cerebral ischemia reperfusion injury.

At present, bone marrow mesenchymal stem cell (BMSC) transplantation has applied to the treatment of cerebral ischemia disease. Nevertheless, the method also has some drawbacks, such as low neural cell conversion rate and weak proliferation ability [37]. Ginsenoside Rg1 is considered to be able to treat cerebral ischemia because its molecules are small enough to pass through the blood-brain barrier [38]. Ischemia reperfusion would cause significant nervous deficit, increase the brain water content, and infarct volume. Interesting, ginsenoside Rg1 can improve nervous deficit, reduce cell apoptosis, and increase the neuron-specific enolase positive cells and glial fibrillary acidic protein positive cells. Furthermore, ginsenoside Rg1 not only significantly increased Bcl-2 protein level, but also decreased Bax protein level. Combined application of ginsenoside Rg1 and BMSC transplantation promoted brain tissue repair through enhancing neuron-like cell differentiation and the antiapoptosis 
effect of ginsenoside Rg1 in cerebral ischemia reperfusion injury [37]. Ginsenoside Rg1 also improved neurological injury via downregulating aquaporin 4 expression [38]. It is well known that inflammation and neuronal apoptosis occur frequently in cerebral ischemia reperfusion injury. Peroxisome proliferator-activated receptor $\gamma(\operatorname{PPAR} \gamma)$ could mediate many signaling pathways in various pathological conditions [72]. Heme oxygenase-1 (HO-1) is one of the downstream effecter of PPAR $\gamma$ [73]. PPAR $\gamma / \mathrm{HO}-1$ signaling can inhibit apoptosis and inflammation. The relationship of ginsenoside Rgl and PPAR $\gamma / \mathrm{HO}-1$ signaling was studied in cerebral ischemia reperfusion injury. They found that the effects of ginsenoside Rg1 and rosiglitazone, the agonist of $\operatorname{PPAR} \gamma$, were similar in activating the $\operatorname{PPAR} \gamma / \mathrm{HO}-1$ signaling. Besides, ginsenoside Rg1 not only decreased the levels of IL$1 \beta$, TNF- $\alpha$, and high-mobility group box-1 (HMGB1), but also downregulated expressions of cleaved caspase-3, cleaved caspase-9, and receptor for advanced glycation end product (RAGE) in model rats [39]. After treatment of ginsenoside Rg1, the brain infarct volume and the permeability of bloodbrain barrier were deceased in ischemia brain. And ginsenoside Rgl would downregulate the protein and mRNA levels of protease-activated receptor-1 (PAR-1). As PAR-1 expression rises, so does blood-brain barrier permeability [40]. What is more, astrocytes play an important role in the ischemic neuronal cell death. Although ginsenoside Rg1 did not change the viability of astrocytes, ginsenoside Rgl could attenuate apoptosis and inhibit intracellular $\mathrm{Ca}^{2+}$ overload in astrocytes. Besides, ginsenoside Rgl could reduce the loss of mitochondrial membrane potential and ROS production in astrocytes [41].

Local inflammation may aggravate cerebral ischemia reperfusion injury. Study showed that ginsenoside Rb1 reversed the activation of nuclear factor kappa $\mathrm{B}(\mathrm{NF}-\mathrm{k} \beta)$ signaling pathway and the elevation of TNF- $\alpha$ and IL- 6 in the ischemic hemisphere [43]. As far as we know, intranasal administration could effectively improve the bioavailability of drug. The concentration of ginsenoside Rb1 could achieve peak concentration at early time after intranasal administration. Moreover, ginsenoside Rbl could inhibit the increase of Beclin 1 level, which is the index of autophagy activity [44]. To explore whether ginsenosides Rg1 and Rb1 have synergistic effect on neuroprotection in ischemia reperfusion, ginsenosides Rg1, Rb1, and Rg1/Rb1 were studied. The results showed that ginsenosides Rg1 and Rb1 significantly improved neurobehavioral deficits via reducing the cell apoptosis and alleviating the lipid peroxidation in the middle cerebral artery occlusion (MCAO) group. Moreover, ginsenosides Rg1 and Rb1 could reverse the decrease of thioredoxin-1 and superoxide dismutase levels, and they could improve the expression of heat shock protein 70 (HSP70), Akt, and the p-NF-kB p65 subunit in the MCAO group. However, ginsenosides Rg1/Rb1 did not have a synergistic effect [45]. Therefore, it may be best to use ginsenosides Rgl or Rbl alone for treating ischemic stroke. In contrast, astragaloside IV combined with ginsenosides Rg1, Rb1, and notoginsenoside R1 have greater effect than those of effective components alone. The combination of four active substrates exerted protective effects on cerebral ischemia injury via antiapoptosis and anti-inflammation. The mechanisms may be associated with inhibiting the activation of NF-k $\beta$, tyrosine kinase 1 /signal transducer, and activator of transcription-1 signal pathways and regulating endoplasmic reticulum stress after cerebral ischemia [42].

Ginsenoside Rd is thought to have neuroprotective effect via antioxidant [74]. Ginsenoside Rd protects against cerebral ischemia through several pathways. Some researches proved that ginsenoside Rd exerted the neuroprotective effect by promoting neurogenesis, increasing vascular endothelial growth factor (VEGF) and BDNF expression, and activating the PI3K/Akt and ERK1/2 pathways [46]. Besides, ginsenoside $\mathrm{Rd}$ can improve behavior score and viability of neurons by inhibiting the hyperactive phosphorylation of $\mathrm{N}$-methyl-D -aspartate receptor 2B (NMDAR 2B) subunit and decreasing its expression levels in cell membrane of ischemia-reperfusion injury models [47]. The content of ginsenoside $\mathrm{Re}$ is the highest among ginsenosides and has good activity. Ginsenoside Re also has protective effect on cerebral ischemia reperfusion injury. Ginsenoside Re exerted the neuroprotection via significantly decreasing the malondialdehyde (MDA) content and increasing the activity of $\mathrm{H}^{+}$-ATPase [48]. Besides, some experiments suggested that ginsenoside $\operatorname{Rg} 3$ modulated various types of ion channels to exert pharmacological effects. The neuroprotective effect of 20(R)-Rg3 was related to attenuating the neuronal apoptosis. The mechanism downregulated the expressions of calpain I and caspase-3 mRNA in hippocampal CA1 region [49] (Table 1).

2.4. Brain Protective Effects against Alzheimer's Disease. Alzheimer's disease (AD) is a neurodegenerative senile dementia that usually occurs in the elderly. The main manifestations are memory loss and cognitive deterioration in the early stage. Etiopathogenesis of $\mathrm{AD}$ is complex, which is mainly related to amyloid $\beta$-proteins $(\mathrm{A} \beta)$ [75]. How to prevent and cure AD becomes a difficult problem. Ginseng has long been used for the prevention and treatment of AD. And heat-processed ginseng had a better effect than raw ginseng in $\mathrm{AD}$ treatment [76].

As we all know, ginsenosides are metabolized into secondary metabolites in the body after oral administration. PPD-type ginsenosides are metabolized to 20-O- $\beta$ D-glucopyranosyl-20(S)-protopanaxadiol (M1) by intestinal bacteria when taken orally.

The cognitive functions of M1 and ginsenoside Rb1 were explored in $\mathrm{AD}$ induced by $\mathrm{A} \beta_{25-35}$ i.c.v. injection. After administration of ginsenosides Rbl and M1, the impaired spatial memory could be improved. The effects of ginsenosides Rb1 and M1 were similar and they could reduce the expression levels of phosphorylated neurofilament- $\mathrm{H}$ and synaptophysin in the cerebral cortex and hippocampus of $\mathrm{AD}$ mice. In addition, M1 had axonal extension activity and did not have effect on dendrites [50]. The study indicated that $\mathrm{AD}$ was closely related to amyloid precursor protein (APP) and presenilin-1 (PS1), which are overexpressed in AD. To explore the anti-AD mechanism of ginsenosides Rg1 and Rg2, a UPLC/MS-based metabolomics investigation was used. The 
results showed that ginsenosides Rg1 and Rg2 reduced the escape latency in Morris water maze compared to the $\mathrm{AD}$ model group. Besides, ginsenosides $\mathrm{Rg} 1$ and $\mathrm{Rg} 2$ decreased $\mathrm{A} \beta_{1-42}$ accumulation in the hippocampus of APP/PS1 mice. In addition, 11 potential biomarkers were detected, which were the metabolism of lysophosphatidylcholines, hypoxanthines, and sphingolipids. This suggested that the anti-AD effect of ginsenosides $\operatorname{Rg} 1$ and $\operatorname{Rg} 2$ would be partly associated with correcting brain metabolic alterations in $\mathrm{AD}$ mice [53]. What is more, ginsenoside Rgl affected lecithin, amino, and sphingolipid metabolism pathways. However, ginsenoside Rb1 affected lecithin and amino acid but not sphingolipid [54]. These findings suggested that metabolomics study might be a new method to find the therapeutic benefits of ginsenosides in $\mathrm{AD}$ treatment.

An $\mathrm{AD}$ transgenic mice was used to exam the protective effect of ginsenoside Rgl on memory performance and synaptic plasticity by using fear conditioning task and western blotting. The results indicated that long-term memory was improved after ginsenoside Rg1 treatment. Ginsenoside Rg1 also did not affect normal diet and weight. Additionally, ginsenoside Rg1 inhibited the levels of C-terminal fragments (CTFs), p-Tau, and $\beta$-amyloid 1-42 in AD mice. Furthermore, ginsenoside Rgl could improve the expression of BDNF and tyrosine receptor kinase $\mathrm{B}(\operatorname{TrkB})$ in the hippocampus. These results indicated that ginsenoside $\mathrm{Rg} 1$ exerted neuroprotective effect by activating the BDNF-TrkB pathway and attenuating the expression of $\mathrm{AD}$-associated proteins [55]. In addition, ginsenoside Rg1 also upregulated the expression of N-methyl-D-aspartate receptor subunit 1 (NR1) and Nmethyl-D-aspartate receptor subunit 2B (NR2B) and slowed the formation of neurofibrillary tangles to improve learning and memory abilities in AD mice [56]. Recently, studies have found that sex hormones, especially estrogen, play an important role in the pathogenesis of $\mathrm{AD}$. The rapid decline of estrogen level in postmenopausal women increases their susceptibility to AD. So, a lack of estrogen may be a risk factor for $\mathrm{AD}$. An AD mice were prepared by ovarian steroid deprivation plus $\mathrm{D}$-gal injection. The results suggested that ginsenoside Rg1 not only restored the impaired cognitive performance and promoted spatial learning and memory, but also reduced the $\mathrm{A} \beta_{1-42}$ production in $\mathrm{AD}$ mice. Besides, ginsenoside Rg1 distinctly increased proportion of $\alpha$-secretase a disintegrin and metallopeptidase domain 10 (ADAM) positive neurons and decreased $\beta$-secretase $\beta$-site APP-cleaving enzyme 1 (BACE) positive neurons in hippocampus of $A D$ mice. Additionally, ginsenoside Rg1 attenuated caspase 3 activity, which also reflected the loss of nerve. Interestingly, ginsenoside Rg1 and 17- $\beta$-estradiol have similar effects on improving cognitive performance and regulating the APP pathway in AD rats [57]. Besides, chronic restraint stress could accelerate the generation and progression of $\mathrm{AD}$. Ginsenoside Rgl protected against ROS oxidative damage and inhibited NOX2, p47phox, and RAC1 expression [58]. The relationship between ginsenoside Rgl and endoplasmic reticulum-stress mediated apoptotic pathway was studied in $\mathrm{AD}$ rats. The results showed that ginsenoside Rgl reduced the accumulation of neurofibrillary tangles and the number of terminal deoxynucleotidyl-transferase-mediated dUTP nick end labeling positive cells in AD mice. What is more, ginsenoside Rgl inhibited the activation of $\mathrm{p}-\mathrm{JNK}$ and reduced the expression of glucose-regulated protein 78 (Grp78). Therefore, ginsenoside Rg1 exhibited neuroprotective effect via blocking the endoplasmic reticulum-stress pathway triggered by inositol-requiring enzyme-1 and tumor necrosis factor receptor-associated factor 2 pathway [59].

Pseudoginsenoside-F11 (PF11), one of the components of ginseng, has been found to have neuroprotection and enhanced neuronal activity [77]. PF11 could decrease escape latency in the Morris water maze and block the reduction of step-through latency in the step-through test on the $\mathrm{A} \beta_{1-42^{-}}$ treated mice and APP/PS1 mice. Besides, PF11 inhibited APP expression and $A \beta_{1-42}$ production in the hippocampus and cortex of APP/PS1 mice. PF11 distinctly increased the activities of SOD and glutathione peroxidase and decreased the amount of MDA. In addition, PF11 downregulated the expression of c-Jun N-terminal protein kinase 2 (JNK 2), p53, and cleaved caspase 3. So, the recognition improvement effect of PF11 might be related to anti-inflammation, antiapoptosis, and the inhibitory effect on amyloidogenesis [60]. Similarly, ginsenoside $\mathrm{R} 1$ was found to have protective effect against $\mathrm{A} \beta$ neurotoxicity [78].

Ginsenoside Rb1 also reduced the $\mathrm{A} \beta_{1-42}$ existence in cortex and hippocampus regions and improved the learning ability of $\mathrm{AD}$ mice. As is known to all, cyclooxygenase2 (COX-2), IkB- $\alpha$, and nNOS are the markers in neuroinflammation. Ginsenoside Rb1 could decrease the COX2 positive cells and IkB- $\alpha$ positive cells and increase the nNOS positive cells in hippocampus compared to model group. So, the neuroprotection effect of ginsenoside Rb1 might be associated with anti-inflammation markers in the hippocampus of AD mice [51]. What is more, ginsenoside Rb1 was absorbed rapidly compared with ginsenosides Re and Rg1 [79]. Therefore, ginsenoside Rb1 may be more effective than other ginsenosides. Besides, ginsenoside Rbl also exhibited a higher scavenging activity against neurotransmitters. For example, ginsenoside Rb1 has a higher scavenging activity against $\mathrm{ONOO}^{-}$than ginsenosides Rb2, Rc, Re, Rg1, and Rg3 [52].

An $\mathrm{AD}$ model was made by injecting aggregated $\beta$ amyloid peptide 1-40 into hippocampus bilaterally. Findings showed that ginsenoside $\mathrm{Rd}$ could ameliorate learning and memory ability and reduce the neuronal death and loss in the CA1 regions of hippocampus in $\mathrm{AD}$ mice. In addition, ginsenoside Rd decreased the expression of Ibal-1, glial fibrillary acidic protein (GFAP), IL- $1 \beta$, IL-6, TNF- $\alpha$, caspase- 3 , and S100 $\beta$ mRNA. Besides, ginsenoside Rd increased IL-10 and HSP70 mRNA expression levels [61]. In addition, ginsenoside $\mathrm{Rd}$ also inhibited the expression of NF-kB p65, which was the important marker of NF-kB pathway [62]. These results indicated the neuroprotective effect of ginsenoside Rd might be associated with anti-inflammation, antioxidation, and antiapoptosis.

Acetylcholinesterase (AChE) and butyrylcholinesterase (BChE) play a significant role in AD. The inhibition of AChE and $\mathrm{BChE}$ provides additional benefits in $\mathrm{AD}$ treatment. Ginsenosides Rb1, Rb2, Rc, Re, Rg1, and Rg3 have significant inhibitory effect against $\mathrm{AChE}$ and BChE. Among these 
TABLE 2: Pharmacological activity and mechanism of ginsenosides on Parkinson and Alzheimer's disease.

\begin{tabular}{|c|c|c|c|}
\hline Pharmacological effects & Ginsenosides & Mechanism & References \\
\hline \multirow[t]{6}{*}{ Anti-Alzheimer's disease } & $\mathrm{Rb} 1$ & $\begin{array}{c}\text { Phosphorylated NF-H and synaptophysin, } \mathrm{ONOO}^{-} \text {, } \\
\mathrm{A} \beta_{1-42}, \mathrm{COX}-2, \mathrm{IkB}-\alpha \downarrow \\
\text { nNOS } \uparrow\end{array}$ & {$[50-52]$} \\
\hline & $\operatorname{Rg} 1$ & $\begin{array}{c}\text { A } \beta_{1-42} \text { accumulation, CTFs, p-Tau, and } \beta \text {-amyloid 1-42, } \\
\text { BACE } 1 \text {, NOX2, p47phox, RAC1, NFTs, } \\
\text { TUNEL-positive cells, p-JNK } \downarrow \\
\text { BDNF, TrkB, NR1, NR2B, ADAM } 10 \uparrow\end{array}$ & {$[53-59]$} \\
\hline & $\mathrm{Rg} 2$ & $\mathrm{~A} \beta_{1-42}$ accumulation & {$[53]$} \\
\hline & F11 & $\begin{array}{c}\mathrm{A} \beta_{1-42} \text { accumulation, } \\
\text { APP expression, MDA, JNK 2, p53, cleaved caspase } 3 \downarrow \\
\text { SOD, GSH-Px } \uparrow\end{array}$ & {$[60]$} \\
\hline & $\mathrm{Rd}$ & $\begin{array}{l}\text { Ibal-1, GFAP, IL-1 } \beta \text {, IL-6, TNF-a, S100 } \beta \text {, IL-10, HSP70, } \\
\text { GSSG/GSH ratio, caspase-3, NF-kB p65 } \downarrow\end{array}$ & {$[61,62]$} \\
\hline & $\mathrm{Re}$ & $\mathrm{AChE} \downarrow$ & {$[52]$} \\
\hline Anti-Parkinson's disease & Rg1 & $\begin{array}{c}\text { MPTP-induced motor, TH, } \\
\text { CD3+CD4+ T cells, } \\
\text { TH, DAT, Bcl-2, Wnt } / \beta \text {-catenin signaling pathway } \uparrow \\
\alpha \text {-synuclein, TNF- } \alpha, \text { IFN- } \gamma \text {, IL-1 } \beta, \text { IL-6, CD3+CD8+ T } \\
\text { cells } \downarrow\end{array}$ & {$[63-68]$} \\
\hline
\end{tabular}

ginsenosides, ginsenoside $\mathrm{Re}$ could better inhibit AChE activity [52]. Some studies found that ginsenosides exerted neuroprotective effects by affecting neurotransmitters in AD. For instance, ginsenosides could increase the levels of $\gamma$-aminobutyric acid, acetylcholine, and dopamine and decrease glutamate and aspartic acid levels in the hippocampus and cortex. Besides, ginsenosides could increase glycine and serotonin levels in the blood [80]. This provides a new way to treat $\mathrm{AD}$ (Table 2).

2.5. Brain Protective Effects against Parkinson's Disease. Parkinson's disease (PD) is a common neurodegenerative disease of the central nervous system, which is characterized by abnormal movements [81]. The typical neuropathy is the degeneration and deletion of dopaminergic neurons in substantia nigra of midbrain, but its pathogenesis is not yet clear. To study the mechanism of ginsenosides in PD is conducive to better treatment of PD.

As is known to all, the most important feature of $\mathrm{PD}$ is movement disorders. Animal models were prepared by using the neurotoxin 1-methyl-4-phenyl-1, 2, 3, 6tetrahydropyridine (MPTP). The accelerating rotarod, wire suspension, and pole tests were used to test the motor behaviors of mice. The results showed that mice treated with MPTP spent more time in wire suspension test and pole test. However, ginsenoside Rg1 alleviates the MPTPinduced motor deficit. Besides, ginsenoside Rgl attenuated the decrease of antityrosine hydroxylase (TH) expression and the increase of $\alpha$-synuclein expression in the substantia nigra and striatum. And ginsenoside Rg1 could alleviate the loss of dopaminergic neurons in treated MPTP mice. So, ginsenoside Rg1 may be a potential therapeutic drug against PD by decreasing $\alpha$-synuclein levels in the SN and striatum $[63,64]$. In addition, ginsenoside Rgl could alleviate the mortality of rats and decrease the level of TNF- $\alpha$, IFN- $\gamma$, IL$1 \beta$, and IL-6 in the substantia nigra pars compacta (SNpc) in MPTP-treated mice $[65,66]$. What is more, T cell subsets would be changed in MPTP-treated mice. Ginsenoside Rg1 not only could increase CD3+CD4+ T cells and percentage of CD4+CD25+Foxp3+ regulatory $\mathrm{T}$ cells, but also decrease $\mathrm{CD} 3+\mathrm{CD} 8+\mathrm{T}$ cells in PD mice. Ginsenoside Rg1 also inhibited the activation of microglia in the SNpc region [66]. 6Hydroxydopamine (6-OHDA) was infused into the medial forebrain bundle to damage the nigrostriatal dopamine pathway of ovariectomized rats. JB-1, the antagonist insulinlike growth factor-I receptor (IGF-IR), and ginsenoside Rg1 were tested in PD model. The finding indicated that ginsenoside Rgl ameliorated the rotational behavior induced by apomorphine, but JB-1 blocked this effect. Additionally, ginsenoside Rgl improved the gene expressions of $\mathrm{TH}$, the dopamine transporter, and $\mathrm{Bcl}-2$ after 6-OHDA lesions, but JB-1 is still against these effects of ginsenoside Rg1. Hence, the effect of Rg1 was closely related to its ability to activate the IGF-IR signaling pathway [67]. Besides, the neuroprotective effects of ginsenoside Rg1 were also blocked by Dickkopf-1, which is antagonist of Wnt signaling pathway. Wnt/ $\beta$-catenin signaling pathways are thought to play an important role in PD [82]. Therefore, the effects of ginsenoside Rg1 were closely related to $\mathrm{Wnt} / \beta$-catenin signaling pathway [68] (Table 2).

\section{Conclusion}

Ginseng is a traditional Chinese medicine. Modern pharmacological studies have shown that it has a regulatory effect on the central nervous system. Ginseng can strengthen the cerebral cortex excitatory and inhibitory processes and reduce the fatigue of the brain process. The protective effect of ginseng is mainly due to the role of ginsenosides. Recently, studies have shown that ginsenosides have effects on the 
nervous system, the cardiovascular system, and the immune system. And few studies have found that ginsenosides are toxic. Although there are many studies on ginsenosides in nervous system diseases, the specific mechanism is still in the basic stage. From what has been discussed above, the neuroprotective mechanism of ginsenosides mainly is mainly related to its anti-inflammatory, antioxidation, antiaging, nerve growth factor, other cytokines, and various signaling pathways. In this paper, we summarize the mechanism of ginsenosides in several neurological diseases in order to explain the neuroprotective mechanism of ginsenoside and provide a useful thinking of ginsenosides in clinical application.

\section{Conflicts of Interest}

All contributing authors declare that there are no conflicts of interest.

\section{Acknowledgments}

This work was supported by Project of Shandong Province Key Research and Development Program (2017YYSP030).

\section{References}

[1] J.-H. Kim, "Cardiovascular diseases and panax ginseng: a review on molecular mechanisms and medical applications," Journal of Ginseng Research, vol. 36, no. 1, pp. 16-26, 2012.

[2] Y. Sun, Y. Liu, and K. Chen, "Roles and mechanisms of ginsenoside in cardiovascular diseases: progress and perspectives," SCIENCE CHINA Life Sciences, vol. 59, no. 3, pp. 292-298, 2016.

[3] Q. Wang, H.-x. Li, L.-m. Liu et al., "The effect Panax ginseng tonic in the treatment of alzheimer's disease," Hebei Medical Journal, vol. 38, no. 22, pp. 3460-3465, 2016.

[4] C.-L. Guo, X.-M. Cui, X.-Y. Yang, and S. Wu, "Advances in studies on biotransformation of ginsensides," Zhongguo Zhong Yao Za Zhi, vol. 39, no. 20, pp. 3899-3904, 2014.

[5] K.-C. Shin and D.-K. Oh, "Characterization of a novel recombinant $\beta$-glucosidase from Sphingopyxis alaskensis that specifically hydrolyzes the outer glucose at the C-3 position in protopanaxadiol-type ginsenosides," Journal of Biotechnology, vol. 172, no. 1, pp. 30-37, 2014.

[6] L. Wang, S.-J. Zhao, Y.-L. Liang, Y. Sun, H.-J. Cao, and Y. Han, "Identification of the protopanaxatriol synthase gene CYP6H for ginsenoside biosynthesis in Panax quinquefolius," Functional \& Integrative Genomics, vol. 14, no. 3, pp. 559-570, 2014.

[7] C.-H. Cui, D. J. Kim, S.-C. Jung, S.-C. Kim, and W.-T. Im, "Enhanced production of gypenoside LXXV using a novel ginsenoside-transforming $\beta$-glucosidase from ginsengcultivating soil bacteria and its anti-cancer property," Molecules, vol. 22 , no. 5, article no. 844, 2017.

[8] W. Li, M.-H. Yan, Y. Liu et al., "Ginsenoside Rg5 ameliorates cisplatin-induced nephrotoxicity in mice through inhibition of inflammation, oxidative stress, and apoptosis," Nutrients, vol. 8, no. 9 , article no. 566, 2016.

[9] C. P. Li, M. S. Zhang, J. Liu et al., "Research of anti-aging mechanism of ginsenoside Rg1 on brain," Zhongguo Zhong Yao Za Zhi, vol. 39, no. 22, pp. 4442-4447, 2014.
[10] S. Tan, F. Zhou, and N. Li, "Anti-fatigue effect of ginsenoside $\mathrm{Rb} 1$ on postoperative fatigue syndrome induced by major small intestinal resection in rat," Biological \& Pharmaceutical Bulletin, vol. 36, no. 10, pp. 1634-1639, 2013.

[11] T. Ahmed, S. H. Raza, and A. Maryam, "Ginsenoside Rb1 as a neuroprotective agent: a review," Brain Research Bulletin, vol. 125, pp. 30-43, 2016.

[12] E. González-Burgos, C. Fernandez-Moriano, and M. P. GómezSerranillos, "Potential Neuroprotective Activity of Ginseng in Parkinson's Disease: A Review," Journal of Neuroimmune Pharmacology, vol. 10, no. 1, pp. 14-29, 2015.

[13] G. Zhang, A. Liu, Y. Zhou, X. San, T. Jin, and Y. Jin, "Panax ginseng ginsenoside- $\mathrm{Rg}_{2}$ protects memory impairment via antiapoptosis in a rat model with vascular dementia," Journal of Ethnopharmacology, vol. 115, no. 3, pp. 441-448, 2007.

[14] W. X. Wang, W. Wang, and K. J. Chen, "Protective effect and mechanism of ginsenosides on central nerve system of animals," Zhongguo Zhong Xi Yi Jie He Za Zhi, vol. 25, no. 1, pp. 189-193, 2005.

[15] F. X. Xu, "Ginsenoside is considered to be of great value in treating epilepsy," Capital Food and Medicine, vol. 22, no. 15, article 21, 2015.

[16] C. Wu, J. Chen, X. Xie, and D. F. Ru, “The influence of Ginseng ginsenosides Rb1 for epilepsy rat hippocampal neurons," The Northern Pharmaceutical, vol. 10, no. 2, pp. 41-42, 2013.

[17] S. L. Moshé, E. Perucca, P. Ryvlin, and T. Tomson, "Epilepsy: New advances," The Lancet, vol. 385, no. 9971, pp. 884-898, 2015.

[18] X.-Y. Lian, Z.-Z. Zhang, and J. L. Stringer, "Anticonvulsant activity of ginseng on seizures induced by chemical convulsants," Epilepsia, vol. 46, no. 1, pp. 15-22, 2005.

[19] X.-Y. Lian, Z. Zhang, and J. L. Stringer, "Anticonvulsant and neuroprotective effects of ginsenosides in rats," Epilepsy Research, vol. 70, no. 2-3, pp. 244-256, 2006.

[20] S. Kim and H. Rhim, "Ginsenosides inhibit NMDA receptormediated epileptic discharges in cultured hippocampal neurons," Archives of Pharmacal Research, vol. 27, no. 5, pp. 524-530, 2004.

[21] E.-J. Shin, Y. H. Koh, A.-Y. Kim et al., "Ginsenosides attenuate kainic acid-induced synaptosomal oxidative stress via stimulation of adenosine A2A receptors in rat hippocampus," Behavioural Brain Research, vol. 197, no. 1, pp. 239-245, 2009.

[22] S. Moussavi, S. Chatterji, E. Verdes, A. Tandon, V. Patel, and B. Ustun, "Depression, chronic diseases, and decrements in health: results from the World Health Surveys," The Lancet, vol. 370, no. 9590, pp. 851-858, 2007.

[23] J.-P. Lépine and M. Briley, "The increasing burden of depression," Neuropsychiatric Disease and Treatment, vol. 7, pp. 3-7, 2011.

[24] M.-L. Wong and J. Licinio, "Research and treatment approaches to depression," Nature Reviews Neuroscience, vol. 2, no. 5, pp. 343-351, 2001.

[25] S. Sohlberg, "Personality, life stress and the course of eating disorders," Acta Psychiatrica Scandinavica, vol. 82, no. s361, pp. 29-33, 1990.

[26] W. Zhu, S. Ma, R. Qu, and D. Kang, "Antidepressant-like effect of saponins extracted from Chaihu-jia-longgu-muli-tang and its possible mechanism," Life Sciences, vol. 79, no. 8, pp. 749-756, 2006.

[27] K. J. Lee and G. E. Ji, "The effect of fermented red ginseng on depression is mediated by lipids," Nutritional Neuroscience, vol. 17, no. 1, pp. 7-15, 2014. 
[28] J. Cui, L. Jiang, and H. Xiang, "Ginsenoside Rb3 exerts antidepressant-like effects in several animal models," Journal of Psychopharmacology, vol. 26, no. 5, pp. 697-713, 2012.

[29] H. Zhang, Z. Li, Z. Zhou, H. Yang, Z. Zhong, and C. Lou, "Antidepressant-like effects of ginsenosides: A comparison of ginsenoside $\mathrm{Rb} 3$ and its four deglycosylated derivatives, $\mathrm{Rg} 3$, $\mathrm{Rh} 2$, compound $\mathrm{K}$, and 20(S)-protopanaxadiol in mice models of despair," Pharmacology Biochemistry \& Behavior, vol. 140, pp. 17-26, 2016.

[30] Z. You, Q. Yao, J. Shen et al., "Antidepressant-like effects of ginsenoside $\mathrm{Rg} 3$ in mice via activation of the hippocampal BDNF signaling cascade," Journal of Natural Medicines, vol. 71, no. 2, pp. 367-379, 2017.

[31] B. Jiang, Z. Xiong, J. Yang et al., "Antidepressant-like effects of ginsenoside Rgl are due to activation of the BDNF signalling pathway and neurogenesis in the hippocampus," British Journal of Pharmacology, vol. 166, no. 6, pp. 1872-1887, 2012.

[32] Z. Liu, Y. Qi, Z. Cheng, X. Zhu, C. Fan, and S. Y. Yu, “The effects of ginsenoside Rg1 on chronic stress induced depression-like behaviors, BDNF expression and the phosphorylation of PKA and CREB in rats," Neuroscience, vol. 322, pp. 358-369, 2016.

[33] B. Lee, I. Shim, H. Lee, and D.-H. Hahm, "Effect of ginsenoside Re on depression- and anxiety-like behaviors and cognition memory deficit induced by repeated immobilization in rats," Journal of Microbiology and Biotechnology, vol. 22, no. 5, pp. 708-720, 2012.

[34] N. Yamada, H. Araki, and H. Yoshimura, "Identification of antidepressant-like ingredients in ginseng root (Panax ginseng C.A. Meyer) using a menopausal depressive-like state in female mice: Participation of 5-HT 2A receptors," Psychopharmacology, vol. 216, no. 4, pp. 589-599, 2011.

[35] X. Wang, C. Zeng, J. Lin et al., "Metabonomics approach to assessing the modulatory effects of St John's Wort, ginsenosides, and clomipramine in experimental depression," Journal of Proteome Research, vol. 11, no. 12, pp. 6223-6230, 2012.

[36] A. Kang, H. Hao, X. Zheng et al., "Peripheral anti-inflammatory effects explain the ginsenosides paradox between poor brain distribution and anti-depression efficacy," Journal of Neuroinflammation, vol. 8, article 100, 2011.

[37] C. Bao, Y. Wang, H. Min et al., "Combination of ginsenoside Rg1 and bone marrow mesenchymal stem cell transplantation in the treatment of cerebral ischemia reperfusion injury in rats," Cellular Physiology and Biochemistry, vol. 37, no. 3, pp. 901-910, 2015.

[38] Y. Zhou, H. Q. Li, and L. Lu, "Ginsenoside Rg1 provides neuroprotection against blood brain barrier disruption and neurological injury in a rat model of cerebral ischemia/reperfusion through downregulation of aquaporin 4 expression," Phytomedicine, vol. 21, no. 7, pp. 998-1003, 2014.

[39] Y. Yang, X. Li, L. Zhang, L. Liu, G. Jing, and H. Cai, “Ginsenoside Rgl suppressed inflammation and neuron apoptosis by activating PPAR $\gamma / \mathrm{HO}-1$ in hippocampus in rat model of cerebral ischemia-reperfusion injury," International Journal of Clinical and Experimental Pathology, vol. 8, no. 3, pp. 2484-2494, 2015.

[40] C.-L. Xie, J.-H. Li, W.-W. Wang, G.-Q. Zheng, and L.-X. Wang, "Neuroprotective effect of ginsenoside-Rg1 on cerebral ischemia/reperfusion injury in rats by downregulating protease-activated receptor-1 expression," Life Sciences, vol. 121, pp. 145-151, 2015.

[41] C. Sun, X. Lai, X. Huang, and Y. Zeng, "Protective effects of ginsenoside Rgl on astrocytes and cerebral ischemic-reperfusion mice," Biological \& Pharmaceutical Bulletin, vol. 37, no. 12, pp. 1891-1898, 2014.

[42] X.-P. Huang, H. Ding, J.-D. Lu, Y.-H. Tang, B.-X. Deng, and C.Q. Deng, "Effects of the combination of the main active components of Astragalus and Panax notoginseng on inflammation and apoptosis of nerve cell after cerebral ischemia-reperfusion," American Journal of Chinese Medicine, vol. 43, no. 7, pp. 14191438, 2015.

[43] J. Zhu, Y. Jiang, L. Wu, T. Lu, G. Xu, and X. Liu, "Suppression of local inflammation contributes to the neuroprotective effect of ginsenoside Rbl in rats with cerebral ischemia," Neuroscience, vol. 202, pp. 342-351, 2012.

[44] T. Lu, Y. Jiang, Z. Zhou et al., "Intranasal ginsenoside Rb1 targets the brain and ameliorates cerebral ischemia/reperfusion injury in rats," Biological \& Pharmaceutical Bulletin, vol. 34, no. 8, pp. 1319-1324, 2011.

[45] X.-S. Zeng, X.-S. Zhou, F.-C. Luo et al., "Comparative analysis of the neuroprotective effects of ginsenosides Rg1 and Rb1 extracted from Panax notoginseng against cerebral ischemia," Canadian Journal of Physiology and Pharmacology, vol. 92, no. 2, pp. 102-108, 2014.

[46] X.-Y. Liu, X.-Y. Zhou, J.-C. Hou et al., "Ginsenoside Rd promotes neurogenesis in rat brain after transient focal cerebral ischemia via activation of PI3K/Akt pathway," Acta Pharmacologica Sinica, vol. 36, no. 4, pp. 421-428, 2015.

[47] Z. Xie, M. Shi, C. Zhang, H. Zhao, H. Hui, and G. Zhao, "Ginsenoside Rd Protects Against Cerebral Ischemia-Reperfusion Injury Via Decreasing the Expression of the NMDA Receptor 2B Subunit and its Phosphorylated Product," Neurochemical Research, vol. 41, no. 8, pp. 2149-2159, 2016.

[48] L.-M. Chen, X.-M. Zhou, Y.-L. Cao, and W.-X. Hu, "Neuroprotection of ginsenoside Re in cerebral ischemia-reperfusion injury in rats," Journal of Asian Natural Products Research, vol. 10, no. 5, pp. 439-445, 2008.

[49] B. He, P. Chen, J. Yang et al., "Neuroprotective effect of 20(R)ginsenoside Rg3 against transient focal cerebral ischemia in rats," Neuroscience Letters, vol. 526, no. 2, pp. 106-111, 2012.

[50] C. Tohda, N. Matsumoto, K. Zou, M. R. Meselhy, and K. Komatsu, "A $\beta(25-35)$-induced memory impairment, axonal atrophy, and synaptic loss are ameliorated by MI, A metabolite of protopanaxadiol-type saponins," Neuropsychopharmacology, vol. 29, no. 5, pp. 860-868, 2004.

[51] Y. Wang, J. Liu, Z. Zhang, P. Bi, Z. Qi, and C. Zhang, "Antineuroinflammation effect of ginsenoside $\mathrm{Rbl}$ in a rat model of Alzheimer disease," Neuroscience Letters, vol. 487, no. 1, pp. 7072, 2011.

[52] R. J. Choi, A. Roy, H. J. Jung et al., "BACE1 molecular docking and anti-Alzheimer's disease activities of ginsenosides," Journal of Ethnopharmacology, vol. 190, pp. 219-230, 2016.

[53] N. Li, Y. Liu, W. Li et al., "A uplc/ms-based metabolomics investigation of the protective effect of ginsenosides rg1 and rg2 in mice with alzheimer's disease," Journal of Ginseng Research, vol. 40, no. 1, pp. 9-17, 2016.

[54] N. Li, L. Zhou, W. Li, Y. Liu, J. Wang, and P. He, "Protective effects of ginsenosides Rg1 and Rb1 on an Alzheimer's disease mouse model: a metabolomics study," Journal of Chromatography B, vol. 985, pp. 54-61, 2015.

[55] F. Li, X. Wu, J. Li, and Q. Niu, "Ginsenoside Rg1 ameliorates hippocampal long-term potentiation and memory in an Alzheimer's disease model," Molecular Medicine Reports, vol.13, no. 6, pp. 4904-4910, 2016. 
[56] X. Li, Y. Liu, X. Zhang, H. Yuan, and Q. Quan, "Effect of ginsenoside Rgl on expressions of phosphory protein Tau and $\mathrm{N}$-methyl-D-aspartate receptor subunits NR1 and NR2B in rat brain slice model of Alzheimer's disease," Zhongguo Zhongyao Zazhi, vol. 35, no. 24, pp. 3339-3343, 2010.

[57] X. Zhang, J. Wang, Y. Xing et al., "Effects of ginsenoside Rg1 or $17 \beta$-estradiol on a cognitively impaired, ovariectomized rat model of Alzheimer's disease," Neuroscience, vol. 220, pp. 191200, 2012.

[58] Y. Wang, H. Kan, Y. Yin et al., "Protective effects of ginsenoside Rg1 on chronic restraint stress induced learning and memory impairments in male mice," Pharmacology Biochemistry \& Behavior, vol. 120, pp. 73-81, 2014.

[59] J.-S. Mu, H. Lin, J.-X. Ye, M. Lin, and X.-P. Cui, "Rg1 exhibits neuroprotective effects by inhibiting the endoplasmic reticulum stress-mediated c-Jun $\mathrm{N}$-terminal protein kinase apoptotic pathway in a rat model of Alzheimer's disease," Molecular Medicine Reports, vol. 12, no. 3, pp. 3862-3868, 2015.

[60] C.-M. Wang, M.-Y. Liu, F. Wang et al., "Anti-amnesic effect of pseudoginsenoside-F11 in two mouse models of Alzheimer's disease," Pharmacology Biochemistry \& Behavior, vol. 106, pp. 57-67, 2013.

[61] J. Liu, X. Yan, L. Li et al., "Ginsennoside Rd attenuates cognitive dysfunction in a rat model of alzheimer's disease," Neurochemical Research, vol. 37, no. 12, pp. 2738-2747, 2012.

[62] J. Liu, X. Yan, L. Li et al., "Ginsenoside Rd Improves Learning and Memory Ability in APP Transgenic Mice," Journal of Molecular Neuroscience, vol. 57, no. 4, pp. 522-528, 2015.

[63] W. Jiang, Z. Wang, Y. Jiang, M. Lu, and X. Li, “Ginsenoside Rg1 ameliorates motor function in an animal model of Parkinson's disease," Pharmacology, vol. 96, no. 1-2, pp. 25-31, 2015.

[64] J. M. Van Kampen, D. B. Baranowski, C. A. Shaw, and D. G. Kay, "Panax ginseng is neuroprotective in a novel progressive model of Parkinson's disease," Experimental Gerontology, vol. 50, no. 1, pp. 95-105, 2014.

[65] Y. Heng, Q.-S. Zhang, Z. Mu, J.-F. Hu, Y.-H. Yuan, and N.H. Chen, "Ginsenoside Rg1 attenuates motor impairment and neuroinflammation in the MPTP-probenecid-induced parkinsonism mouse model by targeting $\alpha$-synuclein abnormalities in the substantia nigra," Toxicology Letters, vol. 243, pp. 7-21, 2016.

[66] T.-T. Zhou, G. Zu, X. Wang et al., "Immunomodulatory and neuroprotective effects of ginsenoside Rg1 in the MPTP(1methyl-4-phenyl-1,2,3,6-tetrahydropyridine) -induced mouse model of Parkinson's disease," International Immunopharmacology, vol. 29, no. 2, pp. 334-343, 2015.

[67] L. Xu, W. F. Chen, and M. S. Wong, "Ginsenoside Rg1 protects dopaminergic neurons in a rat model of Parkinson's disease through the IGF-I receptor signalling pathway," British Journal of Pharmacology, vol. 158, no. 3, pp. 738-748, 2009.

[68] T. Zhou, G. Zu, X. Zhang et al., "Neuroprotective effects of ginsenoside $\operatorname{Rg} 1$ through the Wnt/ $\beta$-catenin signaling pathway in both in vivo and in vitro models of Parkinson's disease," Neuropharmacology, vol. 101, no. 5, pp. 480-489, 2016.

[69] S. L. Livesay, "Clinical review and implications of the guideline for the early management of patients with acute ischemic stroke," AACN Advanced Critical Care, vol. 25, no. 2, pp. 130141, 2014.

[70] K. Strong, C. Mathers, and R. Bonita, "Preventing stroke: saving lives around the world," The Lancet Neurology, vol. 6, no. 2, pp. 182-187, 2007.

[71] V. L. Feigin, C. M. Lawes, D. A. Bennett, S. L. Barker-Collo, and V. Parag, "Worldwide stroke incidence and early case fatality reported in 56 population-based studies: a systematic review," The Lancet Neurology, vol. 8, no. 4, pp. 355-369, 2009.

[72] S. Aleshin, M. Strokin, M. Sergeeva, and G. Reiser, "Peroxisome proliferator-activated receptor (PPAR) $\beta / \delta$, a possible nexus of PPAR $\alpha$ - and PPAR $\gamma$-dependent molecular pathways in neurodegenerative diseases: review and novel hypotheses," Neurochemistry International, vol. 63, no. 4, pp. 322-330, 2013.

[73] S. Y. Park, J. U. Bae, K. W. Hong, and C. D. Kim, "HO-1 Induced by Cilostazol Protects Against TNF- $\alpha$-associated Cytotoxicity via a PPAR- $\gamma$-dependent Pathway in Human Endothelial Cells," Korean Journal of Physiology \& Pharmacology, vol. 15, no. 2, pp. 83-88, 2011.

[74] Y. H. Shang, J. F. Tian, M. Hou, and X. Y. Xu, "Progress on the protective effect of compounds from natural medicines on cerebral ischemia," Chinese Journal of Natural Medicines, vol. 11, no. 6, pp. 588-595, 2013.

[75] J. Hardy and D. J. Selkoe, "The amyloid hypothesis of Alzheimer's disease: progress and problems on the road to therapeutics," Science, vol. 297, no. 5580, pp. 353-356, 2002.

[76] J.-H. Heo, S.-T. Lee, K. Chu et al., "Heat-processed ginseng enhances the cognitive function in patients with moderately severe Alzheimer's disease," Nutritional Neuroscience, vol. 15, no. 6, pp. 278-282, 2012.

[77] Z.-J. Wang, L. Sun, W. Peng et al., "Ginseng derivative ocotillol enhances neuronal activity through increased glutamate release: A possible mechanism underlying increased spontaneous locomotor activity of mice," Neuroscience, vol. 195, pp. 18, 2011.

[78] S. Yan, Z. Li, H. Li, O. Arancio, and W. Zhang, "Notoginsenoside $\mathrm{R} 1$ increases neuronal excitability and ameliorates synaptic and memory dysfunction following amyloid elevation," Scientific Reports, vol. 4, article no. 6352, 2014.

[79] C. Lv, Q. Li, Y. Zhang et al., "A UFLC-MS/MS method with a switching ionization mode for simultaneous quantitation of polygalaxanthone III, four ginsenosides and tumulosic acid in rat plasma: Application to a comparative pharmacokinetic study in normal and Alzheimer's disease rats," Journal of Mass Spectrometry, vol. 48, no. 8, pp. 904-913, 2013.

[80] Y. Zhang, Z. Pi, F. Song, and Z. Liu, "Ginsenosides attenuate $\mathrm{D}$-galactose- and $\mathrm{AlCl} 3$-inducedspatial memory impairment by restoring the dysfunction of the neurotransmitter systems in the rat model of Alzheimer's disease," Journal of Ethnopharmacology, vol. 194, pp. 188-195, 2016.

[81] P. J. G. Ruiz, M. J. Catalán, and J. M. F. Carril, "Initial motor symptoms of Parkinson disease," The Neurologist, vol. 17, no. 6, pp. S18-S20, 2011.

[82] C. L. Parish and L. H. Thompson, "Modulating Wnt signaling to improve cell replacement therapy for Parkinson's disease," Journal of Molecular Cell Biology, vol. 6, no. 1, pp. 54-63, 2014. 


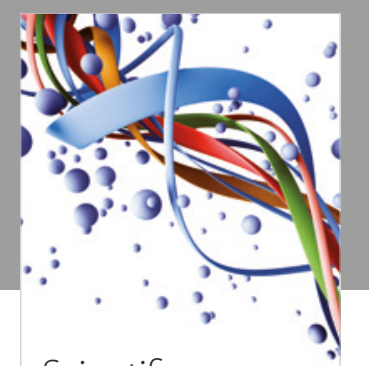

Scientifica
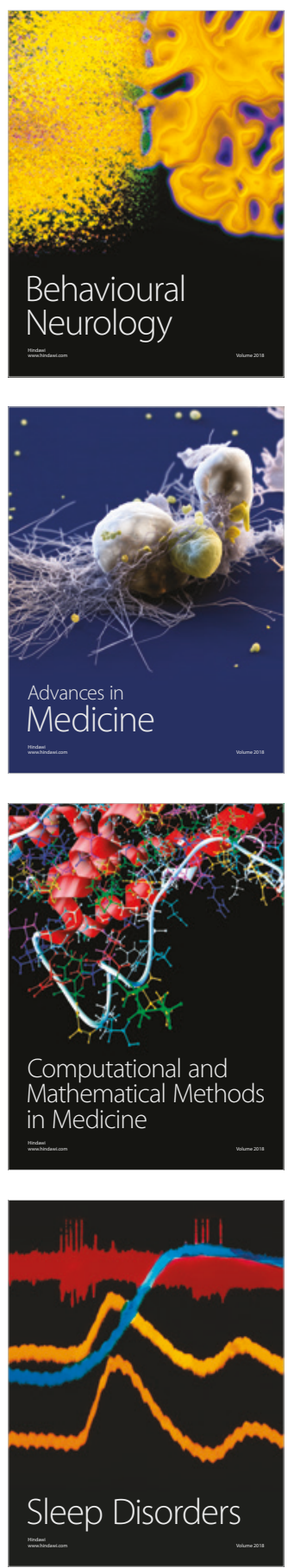

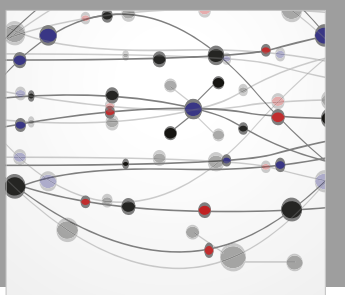

The Scientific World Journal

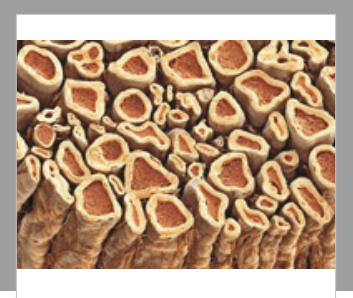

Case Reports in

Neurological Medicine

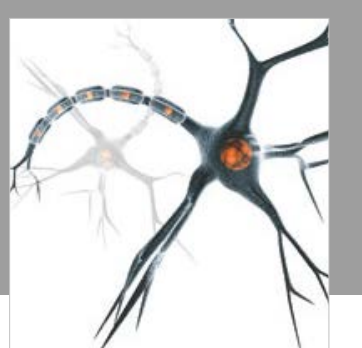

Neural Plasticity

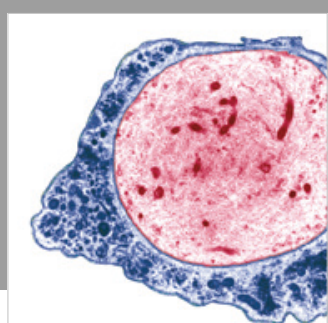

Multiple Sclerosis

International

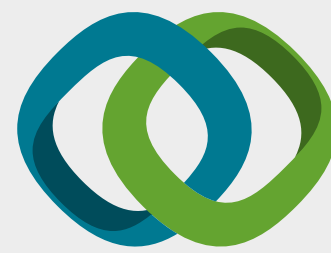

Hindawi

Submit your manuscripts at

www.hindawi.com
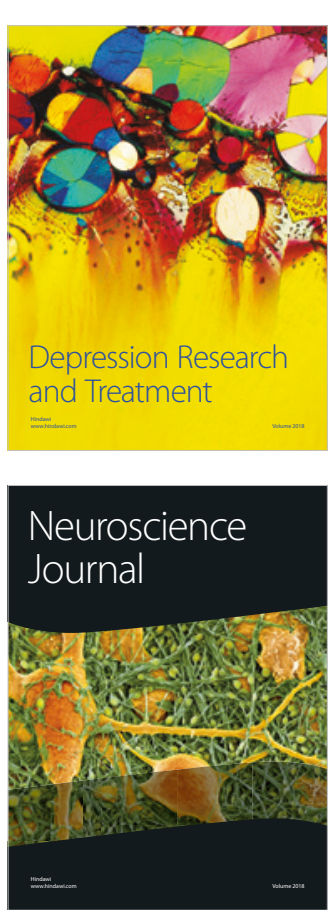

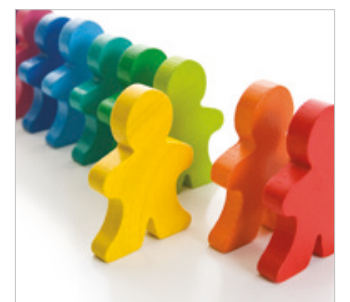

Autism

Research and Treatment
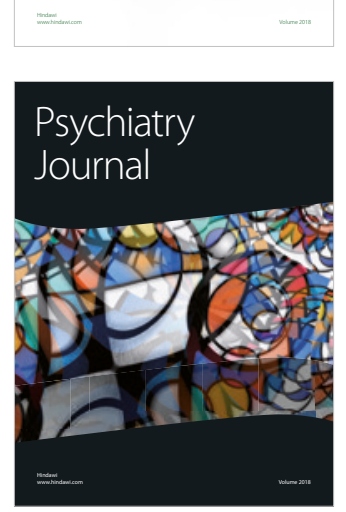
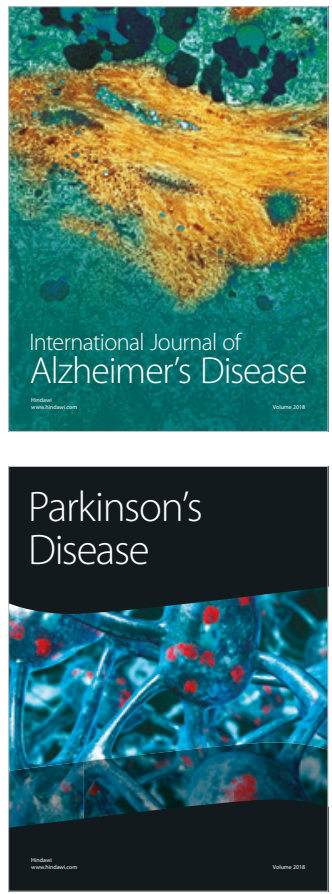
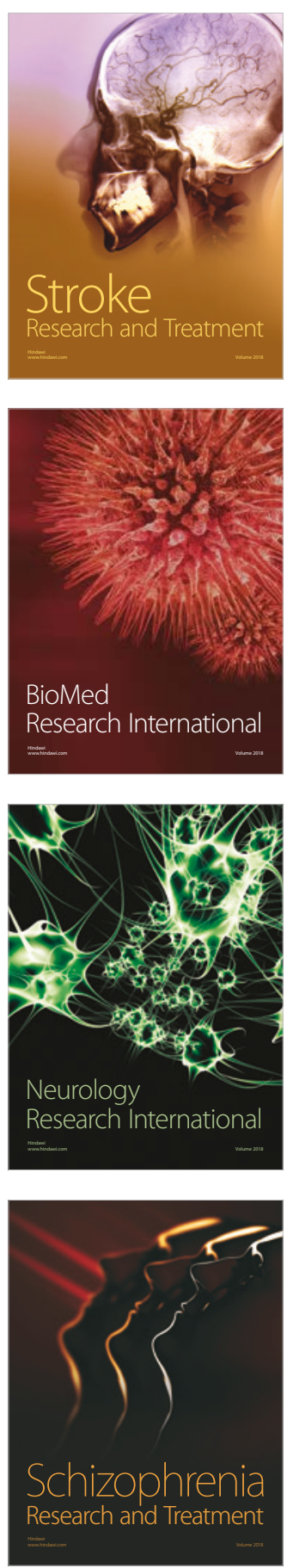